\title{
Is HIV Self-Testing a Potential Answer to the Low Uptake of HIV Testing Services Among Men in Rwanda? Perspectives of Men Attending Tertiary Institutions and Kimisagara Youth Centre in Kigali, Rwanda
}

\author{
Tafadzwa Dzinamarira ${ }^{1,2}$, Gashema Pierre ${ }^{1} \&$ Nadine Rujeni ${ }^{1}$ \\ ${ }^{1}$ College of Medicine and Health Sciences, University of Rwanda, Kigali, Rwanda \\ ${ }^{2}$ Department of Public Health Medicine, School of Nursing and Public Health, University of KwaZulu-Natal, \\ Durban, South Africa \\ Correspondence: Tafadzwa Dzinamarira, College of Medicine and Health Sciences, University of Rwanda, Kigali, \\ Rwanda. ORCID: https://orcid.org/0000-0002-9929-5739. Tel: 250-786-999-838. E-mail: \\ anthonydzina@gmail.com
}

Received: August 21, 2019 Accepted: September 1, 2019 Online Published: September 13, 2019

doi:10.5539/gjhs.v11n11p67

URL: https://doi.org/10.5539/gjhs.v11n11p67

\begin{abstract}
Background and Objective: Rwanda has generally experienced low uptake of HIV testing services among men. However, the reasons behind this have not been researched. The main aim of this study was to explore whether HIV self-testing (HIVST) would have the capacity to improve uptake of HIV testing services among men in Rwanda.

Methods: We conducted a qualitative study of 22 men attending tertiary institutions and the Kimisagara Youth Centre in Kigali, Rwanda. Data collection was conducted through open interviews. Data analysis was conducted through thematic content analysis.

Results: Our findings revealed that most men had poor knowledge of HIVST, but the majority were willing to adopt it. Four main themes emerged during data analysis. Theme one indicated that men experienced a lack of sufficient information on HIVST. From theme two, it was uncovered that some men were indifferent to HIVST. From theme three, it emerged that most men perceived the cost as the main barrier to HIVST; however, if it was offered free of charge, they were willing to adopt it. Finally, theme four revealed that most men willing to adopt HIVST were concerned about the potential social harm and possible adverse events associated with HIVST.
\end{abstract}

Keywords: HIV self-testing, men, perception, Rwanda

\section{Introduction}

Men are said to be less likely to seek out health care and be tested for the human immunodeficiency virus (HIV) (UNAIDS, 2017). This pattern could be attributed to masculinity, poor service delivery, long waiting times to obtain the results, concerns over confidentiality and HIV-related stigma (Addis \& Mahalik, 2003; Kojima \& Klausner, 2018; REN et al., 2017). Current HIV testing services (HTS) are also perceived by men as unwelcoming (Dzinamarira \& Mashamba-Thompson, 2019; Kojima \& Klausner, 2018). Consequently, many new HIV infections go unrecognized for extended periods of time.

HIV self-testing (HIVST) is an emerging low-cost screening approach with the potential to overcome barriers associated with traditional HIV testing by allowing individuals to test themselves in a private setting at their convenience (Figueroa et al., 2018; Volk et al., 2016; WHO, 2016). Apart from being reliable, safe and accurate, this approach empowers those who may not otherwise be tested, helps increase serostatus awareness and treatment initiation and ultimately represents a way to deliver preventive services to hard-to-reach populations (Carmen Figueroa, Johnson, Verster, Baggaley, \& Behavior, 2015; Kojima \& Klausner, 2018).

Although HIV testing has served as a cornerstone in the fight against the HIV epidemic, available evidence shows low rates of HIV testing among men in Rwanda (National Institute of Statistics of Rwanda, 2016). Progress has been made to improve HTS; however, according to the Rwanda 2015 Demographic Health Survey, 24\% of men 
have never had an HIV test, and there is an even higher percentage of youth aged 15 to 24 who have never been tested $(67.2 \%)$. HIVST has the potential to overcome barriers associated with HIV testing by allowing individuals to test themselves in a private setting at their own convenience. A few sub-Saharan African countries, for example, Kenya, have already implemented HIVST, whereas other countries are considering introducing it as part of their national strategic plans, testing strategies, policies and regulatory frameworks. HIVST has been shown to be acceptable testing strategy in a variety of populations globally. There is a paucity of data in Rwanda; this study, therefore, focused on the perspectives of male youth toward HIVST.

\section{Methods}

\subsection{Setting and Study Population}

A trained health professional, GP, conducted the interviews in a private room at each of the study sites. We purposively selected one public youth center, one private university and one public university as study sites. Our study population included men attending the selected sites.

\subsection{Sampling and Eligibility}

A convenience sampling method was used to recruit participants. Men who self-reported being unaware of their HIV status at the time of the study were invited to participate. Our participants were routinely attending the study sites. The sample size was determined by saturation of the data (Saunders et al., 2018).

\subsection{Data Collection}

Data collection was performed using a phenomenological approach through in-depth, open semistructured interviews (Streubert Speziale \& Carpenter, 2003). Interviews lasted between 20 and 40 minutes. The researchers developed the interview guide based on previously published research questions (Dodds et al., 2018; Freeman et al., 2018; WHO, 2018). We piloted our interview guide with two participants to ensure dependability (Holloway, 2005; Polit \& Beck, 2008). The interview guide was revised prior to study commencement.

All interviews were tape-recorded, and interviews were transcribed word for word by a professional transcriber. The transcribed text from each informant was translated from Kinyarwanda to English. The first and second authors read all the transcripts while listening to the audiotapes to ensure the accuracy of the transcription. Reading and coding were initiated while the data were being collected. Along with the observation notes taken during each interview, the transcripts were coded for thematic content analysis. Using the storyline as a guideline, subsidiary categories were related both to the core category and to each other at their dimensional level. In the end, those relationships were validated against the data. Finally, an overall interpretation was made to explain how thematic areas related to one another and how the various concepts related to the study question. We employed NVivo (QSR International Pty Ltd. Version 12, 2018.) qualitative data analysis software.

\subsection{Trustworthiness of Qualitative Data}

During the interview process, we used prolonged engagement to ensure credibility (Lincoln \& Guba, 1985; Talbot, 1995). Data analysis was based on the naturalistic paradigm with a conventional content analysis in which coding categories were derived directly from the text data (Fereday \& Muir-Cochrane, 2006). This approach has been shown to limit researcher bias due to preconceived ideas or other theoretical perspectives.

\section{Results}

Theme one indicated that men experienced a lack of sufficient information on HIVST. Their perceived knowledge was poor, with less than half of the participants knowing the details of the HIVST. Responses from study participants indicated that they were drawing a conclusion on HIVST based on the name of the test process that implies one has to test themselves to obtain an HIV result. Participants' responses indicated that they had insufficient understanding of HIVST. From their responses, the subtheme that health care institutions were severely lacking in information, education, and communication (IEC) materials, and other resources concerning HIVST emerged. The participant responses indicated that they were not informed about HIVST on their visits to health care institutions for routine healthcare.

The perceived knowledge of HIV self-testing varied by age group. Younger men were interested in trying out a new test that they described as a comfortable option:

I would definitely try out this test as I think it is comfortable. There is no harm associated with the collection of blood or finger-prick as with current tests available at health facilities. (18-year-old, does not know HIV serostatus).

At the individual level, perceived knowledge of HIV self-testing was mainly obtained from reading the local 
newspaper and included general information on specimen type and how long one would need to wait for results:

I read about it [HIV self-testing] in the newspaper some time back. They say it can work even on saliva and gives results in ten minutes. (25-year-old, does not know HIV serostatus).

From theme two, it was uncovered that some men were indifferent to HIVST. They were not interested in adopting it or knowing their HIV status. Interestingly, a subtheme emerged indicating that some men saw HIV testing as a woman's responsibility. They indicated that they would infer their HIV status from their spouse or partner's status:

I have never tested for HIV my whole life. But I am still healthy. I won't say that I will not try HIV self-testing, but I also am not sure I will try it.... (29-year-old, does not know HIV serostatus).

......I know my wife will get tested. If she comes [with a] negative [HIV result], then I know I am negative. My wife is as good as me. If she is [HIV] negative then so am I. (27-year-old, does not know HIV serostatus).

From theme three, it emerged that most men perceived cost as the main barrier to HIVST but that, if it were offered free of charge, they were willing to adopt it. Men who were willing to adopt HIVST cited reasons such as confidentiality, convenience, fear of stigma, and privacy as reasons for acceptability of HIVST.

You know Rwanda is very small. If you go to the health center even to the big hospitals, you are most likely going to find someone you know working in those clinics. For some of us we even went to school with the staff there or they know our brothers and sisters. Self-testing will allow me to avoid all those people and just test when at home. I will use it. I like it. I just hope it will be offered for free maybe at places like condom kiosks (25-year-old, does not know HIV serostatus)

Theme four indicated that most men willing to adopt HIVST were concerned about the potential social harm and possible adverse events associated with HIVST. Men expressed concern over the perceived social harm associated with the lack of immediate post-test counseling.

...there are a lot; depression, if I tested myself and I find I am HIV positive, it will take me time to mention this to everyone around me, and this will lead to depression and even to suicide. I think that can be a harmful impact of this test. (27-year-old, does not know HIV serostatus).

The worse thing that would happen is to cause someone to have trauma, kill him/herself once you find out that you are HIV positive. .... For [HIV] self-testing because there is nobody behind you, [this] would be the reason, and [at] the clinic, [this] would happen rarely because it [HIV testing] is followed by counseling and other guidance that helps you to accept yourself and ways to get medicine. (30-year-old, does not know HIV serostatus).

\section{Discussion}

Various scholars have carried out different studies concerning HIVST, and they have provided mixed results. While there have been considerable efforts aimed at promoting the knowledge of HIV status, half of all individuals who live with HIV are still not aware of their HIV status (Gumede \& Sibiya, 2018). There is need ensuring that adequate information concerning the use of HIVST is provided.

In tandem with the findings of the current study, Witzel et al., indicated that HIVST kits and instructions are unnecessarily complicated and did not cater to the range of abilities of those intended to use them (Witzel, Rodger, Burns, Rhodes, \& Weatherburn, 2016).

The lack of adequate HIVST information is one of the main challenges that hinder adoption of HIVST (Tonen-Wolyec et al., 2018). A lack of information also makes users more likely to misinterpret the test results. Therefore, the scholars recommended supervised use of HIVST among the people who are poorly educated (Tonen-Wolyec et al., 2018).

Similar to this study, the findings of other scholars (Grésenguet, de Dieu Longo, Tonen-Wolyec, Bouassa, \& Belec, 2017; van Rooyen et al., 2015) revealed a lack of information concerning the use of HIVST. The researchers noted the need for educational pictograms and notices to be in the vernacular language(s) to avoid the frequent difficulties faced with the use of HIVST and the frequent misinterpretation of the test results (Grésenguet et al., 2017).

A different study revealed that the use of HIVST ensured that there was privacy as well as confidentiality (Moyo \& Mokgatle, 2016). On the other hand, other scholars have reported cost of test kits as a barrier for uptake (Frye et al., 2015; Katz, Golden, Hughes, Farquhar, \& Stekler, 2012). The other notable challenges that were pointed out by these researchers include the general lack of posttest counseling and the general lack of strategies to connect the self-test results to HIV programming. They noted the need for the provision of clear instructions on how individuals can test themselves. This is a concern that has also been addressed by the present study. 
The cost and cost-effectiveness of HIVST have been a matter of concern (Huang et al., 2015; Maheswaran et al., 2016). When evaluating the cost associated with HIVST, it is highly critical to take into consideration not only the unit price for every test kit but also the financial impact of different HIVST approaches (Huang et al., 2015; Maheswaran et al., 2016). Based on a mathematical model built on data obtained from Zimbabwe, HIVST may be highly cost-effective when the test kits cost approximately $\$ 3$ US per unit and when there is a moderate (20\%) increase in HIV testing because of HIVST (Cambiano et al., 2015). It is probable that HIVST will be highly cost-effective within settings that have lower testing coverage when options for support after positive self-testing increases; when HIV-negative people link with HIV prevention programs, such as VMMC and PrEP; and when people at high ongoing risk of HIV increase their testing frequency.

Our participants expressed concern over the perceived social harm associated with the lack of immediate posttest counseling. This observation contradicts the findings of studies conducted by (Brown, Djimeu, \& Cameron, 2014; Carmen Figueroa et al., 2015), which established that there is generally no clear evidence supporting adverse events, such as inter-partner violence, adverse emotional reactions to positive tests, forced/coerced testing, mental health or psychosocial issues, and suicide or self-harm, due to HIVST.

\section{Conclusion}

Issues including the lack of knowledge and the lack of pre- and posttest counseling seemed to be issues of concern that must be addressed if HIVST is to be successfully implemented among men in Rwanda. There is a need to provide health facilities with HIVST information and IEC materials necessary to maximize mobilization and ultimately uptake.

\subsection{Limitations}

As with other qualitative studies, the quality of data is highly subjective. However, we used a rigorous thematic content analysis process where data were generated directly from participant responses.

\section{Declarations}

\section{Ethics Approval and consent to participate}

This study was approved by the University of Rwanda, College of Medicine and Health Sciences Institutional Review Board (approval number: 094/CMHS IRB/2018). Permission was obtained from the Principals at the tertiary institutions and the Coordinator at Kimisagara Youth Centre prior to conducting this study. All study participants also signed an informed consent form prior to participating in the study.

\section{Consent to Publish}

Not applicable

\section{Availability of Data and Materials}

If needed, the interview transcripts used for this article are available upon reasonable request in writing to the corresponding author. Permission will need to be sought from University of Rwanda, College of Medicine and Health Sciences Institutional Review Board.

\section{Funding}

No funding was received for this study.

\section{Acknowledgements}

We thank all the individuals who took time to participate in the interviews for this study. This study would have not been possible without support from the University of Rwanda, College of Medicine and Health Sciences.

\section{Competing Interests Statement}

The authors declare that they have no competing interests that may have inappropriately influenced them in writing this article

\section{References}

Addis, M. E., \& Mahalik, J. R. J. A. p. (2003). Men, masculinity, and the contexts of help seeking. American Psychologist, 58(1), 5. https://doi.org/10.1037/0003-066X.58.1.5

Brown, A. N., Djimeu, E. W., \& Cameron, D. B. (2014). A review of the evidence of harm from self-tests. AIDS and Behavior, 18(4), 445-449. https://doi.org/10.1007/s10461-014-0831-y

Cambiano, V., Ford, D., Mabugu, T., Napierala Mavedzenge, S., Miners, A., Mugurungi, O., . . Phillips, A. (2015). Assessment of the potential impact and cost-effectiveness of self-testing for HIV in low-income countries. 
The Journal of infectious diseases, 212(4), 570-577. https://doi.org/10.1093/infdis/jiv040

Dodds, C., Mugweni, E., Phillips, G., Park, C., Young, I., Fakoya, F., .. Chwaula, J. (2018). Acceptability of HIV self-sampling kits (TINY vial) among people of black African ethnicity in the UK: a qualitative study. BMC Public Health, 18(1), 499. https://doi.org/10.1186/s12889-018-5775-0

Dzinamarira T, Mashamba-Thompson TP. Factors Contributing Toward Men's Engagement With HIV Services: A Narrative Review. Global Journal of Health Science [Internet]. Canadian Center of Science and Education; 2019 Aug 22;11(10):150. Available from: http://dx.doi.org/10.5539/gjhs.v11n10p150

Fereday, J., \& Muir-Cochrane, E. (2006). Demonstrating rigor using thematic analysis: A hybrid approach of inductive and deductive coding and theme development. International journal of qualitative methods, 5(1), 80-92. https://doi.org/10.1177/160940690600500107

Figueroa, C., Johnson, C., Ford, N., Sands, A., Dalal, S., Meurant, R., . . Baggaley, R. (2018). Reliability of HIV rapid diagnostic tests for self-testing compared with testing by health-care workers: a systematic review and meta-analysis. Lancet HIV, 5(6), e277-e290. https://doi.org/10.1016/S2352-3018(18)30044-4

Figueroa, C., Johnson, C., Verster, A., Baggaley, R. J. A., \& behavior. (2015). Attitudes and acceptability on HIV self-testing among key populations: a literature review. AIDS and Behavior, 19(11), 1949-1965. https://doi.org/10.1007/s10461-015-1097-8

Freeman, A. E., Sullivan, P., Higa, D., Sharma, A., MacGowan, R., Hirshfield, S., . . Prevention. (2018). Perceptions of HIV Self-Testing Among Men Who Have Sex With Men in the United States: A Qualitative Analysis. AIDS Education and Prevention, 30(1), 47-62. https://doi.org/10.1521/aeap.2018.30.1.47

Frye, V., Wilton, L., Hirshfield, S., Chiasson, M. A., Usher, D., Lucy, D., . . Team, A. A. M. S. (2015). "Just because it's out there, people aren't going to use it." HIV self-testing among young, Black MSM, and transgender women. AIDS patient care and STDs, 29(11), 617-624. https://doi.org/10.1089/apc.2015.0100

Grésenguet, G., de Dieu Longo, J., Tonen-Wolyec, S., Bouassa, R.-S. M., \& Belec, L. (2017). Acceptability and usability evaluation of finger-stick whole blood HIV self-test as an HIV screening tool adapted to the general public in the Central African Republic. The open AIDS journal, 11, 101. https://doi.org/10.2174/1874613601711010101

Gumede, S. D., \& Sibiya, M. N. (2018). Health care users' knowledge, attitudes and perceptions of HIV self-testing at selected gateway clinics at eThekwini district, KwaZulu-Natal province, South Africa. SAHARA-J: Journal of Social Aspects of HIV/AIDS, 15(1), 103-109. https://doi.org/10.1080/17290376.2018.1517607

Holloway, I. (2005). Qualitative research in health care: McGraw-Hill Education (UK).

Huang, E., Marlin, R., Medline, A., Young, S., Daniels, J., \& Klausner, J. (2015). P17. 09 Cost-effectiveness of hiv self-testing promotion through grindr ${ }^{\mathrm{TM}}$, a smartphone social networking application. In: BMJ Publishing Group Ltd. https://doi.org/10.1136/sextrans-2015-052270.587

Katz, D. A., Golden, M. R., Hughes, J. P., Farquhar, C., \& Stekler, J. (2012). Acceptability and ease of use of home self-testing for HIV among men who have sex with men. Paper presented at the 19th Conference on Retroviruses and Opportunistic Infections. Seattle, WA.

Kojima, N., \& Klausner, J. D. (2018). Accelerating epidemic control: the role of HIV self-testing. The lancet HIV. https://doi.org/10.1016/S2352-3018(18)30063-8

Lincoln, Y. S., \& Guba, E. G. (1985). Establishing trustworthiness. Naturalistic inquiry, 289, 331.

Maheswaran, H., Petrou, S., MacPherson, P., Choko, A. T., Kumwenda, F., Lalloo, D. G., . . Corbett, E. L. (2016). Cost and quality of life analysis of HIV self-testing and facility-based HIV testing and counselling in Blantyre, Malawi. BMC medicine, 14(1), 34. https://doi.org/10.1186/s12916-016-0577-7

Moyo, T., \& Mokgatle, M. (2016). Opinions about and acceptability of HIV self-testing amongst students at the Institute of Health Sciences-Lobatse, Botswana. Sefako Makgatho Health Sciences University,

National Institute of Statistics of Rwanda, M. o. F. a. E. P. R., Ministry of Health/Rwanda, and ICF International. (2016). Rwanda Demographic and Health Survey 2014-15. Kigali, Rwanda. Retrieved from http://dhsprogram.com/pubs/pdf/FR316/FR316.pdf

Polit, D. F., \& Beck, C. T. (2008). Nursing research: Generating and assessing evidence for nursing practice. Lippincott Williams \& Wilkins. 
Ren, X. L., Wu, Z. Y., Mi, G. D., Mcgoogan, J., Rou, K. M., Yan, Z. J. B., \& Zhang, E. (2017). Uptake of HIV self-testing among men who have sex with men in Beijing, China: a cross-sectional study. Infectious Diseases of Poverty, 6(1). https://doi.org/10.1186/s40249-017-0326-y

Saunders, B., Sim, J., Kingstone, T., Baker, S., Waterfield, J., Bartlam, B., . . . Jinks, C. (2018). Saturation in qualitative research: exploring its conceptualization and operationalization. Quality \& quantity, 52(4), 1893-1907. https://doi.org/10.1007/s11135-017-0574-8

Streubert Speziale, H., \& Carpenter, D. (2003). Qualitative Research in Nursing (3rd ed.). Philadelphia USA. Lippincott Williams \& Wilkins.

Talbot, L. A. (1995). Principles and practice of nursing research, Mosby St. In: Louis.

Tonen-Wolyec, S., Batina-Agasa, S., Muwonga, J., N'kulu, F. F., Bouassa, R.-S. M., \& Bélec, L. (2018). Evaluation of the practicability and virological performance of finger-stick whole-blood HIV self-testing in French-speaking sub-Saharan Africa. PloS one, 13(1), e0189475. https://doi.org/10.1371/journal.pone.0189475

UNAIDS. (2017). Blind spot - Reaching out to men and boys addressing a blind spot in the response to HIV.

van Rooyen, H., Tulloch, O., Mukoma, W., Makusha, T., Chepuka, L., Knight, L. C., . . . Chirwa, E. (2015). What are the constraints and opportunities for HIVST scale-up in Africa? Evidence from Kenya, Malawi and South Africa. Journal of the International AIDS Society, 18(1), 19445. https://doi.org/10.7448/IAS.18.1.19445

Volk, J. E., Lippman, S. A., Grinsztejn, B., Lama, J. R., Fernandes, N. M., Gonzales, P., . . Buchbinder, S. (2016). Acceptability and feasibility of HIV self-testing among men who have sex with men in Peru and Brazil. International journal of STD \& AIDS, 27(7), 531-536. https://doi.org/10.1177/0956462415586676

World Health Organization [WHO]. (2016). Guidelines on HIV self-testing and partner notification: supplement to consolidated guidelines on HIV testing services (9241549866).

World Health Organization [WHO]. (2018). HIV Self-Testing Strategic Framework: A Guide For Planning, Introducing And Scaling Up HIV Testing Services. Retrieved from Geneva, Switzerland: https://apps.who.int/iris/bitstream/handle/10665/275521/9789241514859-eng.pdf?ua=1

Witzel, T. C., Rodger, A. J., Burns, F. M., Rhodes, T., \& Weatherburn, P. J. P. O. (2016). HIV self-testing among men who have sex with men (MSM) in the UK: a qualitative study of barriers and facilitators, intervention preferences and perceived impacts. Plos one, 11(9), e0162713. https://doi.org/10.1371/journal.pone.0162713

\section{Copyrights}

Copyright for this article is retained by the author(s), with first publication rights granted to the journal.

This is an open-access article distributed under the terms and conditions of the Creative Commons Attribution license (http://creativecommons.org/licenses/by/4.0/). 